\title{
A Thermal conductivity and electromotive force measurement system for nuclear fuels and materials
}

\author{
Changhu Xing ${ }^{1}$, Colby Jensen ${ }^{2}$, Charles Folsom ${ }^{1}$, Heng Ban ${ }^{1}$, and J. Rory Kennedy ${ }^{2}$ \\ ${ }^{1}$ Mechanical \& Aerospace Engineering Department, Utah State University, Logan, UT 84322-4130 \\ ${ }^{2}$ Idaho National Laboratory, Idaho Falls, ID 83415 \\ Email: changhu.xing@aggiemail.usu.edu; heng.ban@usu.edu
}

\begin{abstract}
The development of advanced nuclear fuels requires a better understanding of their transmutation and microstructural evolution. Alloy fuels have the advantage of high thermal conductivity and improved characteristics in fuel-cladding chemical reaction. However, information on thermodynamic and thermophysical properties is limited. The objective of this research is to develop an experimental system, integrated with the thermal conductivity measurement by the comparative cut-bar technique to measure the thermodynamic properties of solid materials, from which an understanding of their phase change(s) can be determined. With the coupled system, thermal conductivity and electromotive force (EMF) can both be measured. In order to calibrate and test the system, the apparatus was employed to measure the EMF of several materials. As an initial calibration test, the EMF of Chromel was measured from $100{ }^{\circ} \mathrm{C}$ to $800{ }^{\circ} \mathrm{C}$ and compared with theoretical values. The subsequent material structure determination process was performed by EMF measurement of pure iron, iron-nickel alloy and ANSI 1018 carbon steel rods. The measured phase transition temperatures were compared with the corresponding alloy equilibrium phase diagrams. The results indicate that the system is able to determine material phase change based on EMF measurement. In the future, this prototype system is to be eventually modified and used in a hot-cell on irradiated samples.
\end{abstract}

\section{Keywords}

Electromotive force, material structure, phase transition, thermopower.

\section{Introduction}

Advanced nuclear fuels are essential to transmute highly radioactive and long lived transuranic isotopes in order to close the nuclear fuel cycle. Integral to the implementation of the fuel development is an understanding of the performance and behavior of transmuted nuclear fuels, mainly, the material structure of the alloy fuels. The methods for revealing material phase diagrams may be categorized as either computational or experimental. While the computational method (CALPHAD) [1] predicts/extrapolates the phase equilibria by applying Gibbs energy minimization, the experimental techniques are the foundation for the computation and provide inputs for the optimization of the phase diagram.

Many experimental techniques can be used to determine the thermodynamic properties of a material such as calorimetric measurement [2], gas phase equilibria techniques [3] and electromotive force measurement [4]. Among these methods, the EMF measurement technique has unique advantages. When a thermal cell is built, it involves a direct measurement of system EMF and Seebeck coefficient (thermopower or thermoelectric power) of a sample material when it is contacted with a reference conductor whose thermoelectric properties are known. When a sample material undergoes a pressure induced or temperature led phase transformation, both its structural and electronic properties change correspondingly. The Seebeck coefficient generally presents an anomalous behavior in the form of an abrupt variation corresponding to the change of material structure [5-11].

Another advantage for the EMF measurement adopted in this research is the integrated measurements with thermal conductivity. An advanced comparative axial heat flow technique for thermal conductivity measurement has been developed in this laboratory [12-15]. With the available system, the thermal EMF can be measured directly without incurring any additional cost and equipment. Compared to other measurement systems, the overall system design is unique to provide possible measurement of both thermal conductivity and specimen EMF up to high 
temperatures $\left(\sim 1000^{\circ} \mathrm{C}\right)$. The results provided in this paper demonstrate the utility of the unique design approach where control of the temperature gradient is provided by the natural gradient in the tube furnace, a radiative heat sink, and small heaters placed in the assembly.

The objective of this study is to describe a laboratory-based experimental system for the determination of material thermodynamic properties. The system is to be combined with thermal conductivity measurements and used in a controlled environment such as a glove box or radioactive hot cell. Calibration of the system using $\mathrm{N}$ type and $\mathrm{S}$ type thermocouple (TC) elements as reference materials was performed with respect to several samples. A calibration test on a material with no phase transition was done using a Chromel wire, one leg of the K type TC. Tests performed on materials with phase change(s) in their solid state include: (i) pure iron, a pure element; (ii) ironnickel alloy, a binary alloy; and (iii) ANSI 1018 carbon steel, a multiple component alloy. The measurement system will be adapted for the determination of material structure of alloy fuels at a later stage.

\section{Measurement principle and system}

An electric potential (EMF) is generated in a metal or semiconductor when there is a temperature difference. The Seebeck coefficient $\left[\mathrm{VK}^{-1}\right]$, a measure of the voltage produced with a unit temperature difference is expressed as $S=\Delta V / \Delta T$ where $\Delta V$ is the measured EMF [V] and $\Delta T$ is the temperature difference [K]. The EMF depends only on material and temperature difference but not on the dimension of the conductor.

The thermopower determination is usually conducted in comparison to a reference because the leads to the voltmeter generate another EMF. Figure 1 presents an illustration of the configuration for thermopower determination [16], with the help of TCs. Two TCs are injunction with the unknown material $X$, with a temperature difference $\Delta T$ in between. The circuit EMF, $\Delta V_{X R}$ measured from either one of the two TC leads, is attributed to the combined potential difference of two materials (reference and unknown). The thermopower of the unknown material, $S_{X}$, can be expressed as

$$
S_{X}=S_{R}-\Delta V_{X R} / \Delta T
$$

where $S_{R}$ is the thermopower of the reference material whose value is taken from Ref. [17] with the addition of the absolute thermopower of platinum $[18,19]$. The measured sample thermopower is usually considered as the material property at the mean sample temperature $T+\Delta T / 2$ [20]. The magnitude of temperature difference, $\Delta T$, is limited by the desired accuracy of thermopower in Eq. 1 and the resolution and precision of the equipment used in determining the temperature and electric potential. The applied temperature difference is typically in the range of 2-5 K [20, 21].

The measurement system, which was used for thermal conductivity measurement by comparative axial heat flow method, is employed in the EMF measurement (Fig. 2). Detailed description of the system and thermal conductivity measurements of unknown samples are described elsewhere [12-15]. This paper presents only an introduction of the system and results of the EMF measurement side.

The sample and reference bars are surrounded by insulation material to minimize lateral heat loss, both then being enclosed by the guard. Heaters on one side increase the temperature of the test stack and guard and heat sink on the other side cools the unit. An axial temperature gradient is created allowing the thermal conductivity determination by the TC temperature measurements. The system is placed in a MTI OTF-1200X tube furnace to get the required ambient temperature, and the desired temperature differences in the sample and reference bars are built up and maintained by the heaters, driven by a dual-loop Eurotherm 3504 controller. Inert gas is backfilled into the tube furnace to protect the measurement system after the air is purged by a vacuum pump.

Thermal conductivity is measured at intermittent temperatures because of the steady state requirement. The EMF measurement, however, is conducted continuously in a quasi-static way. With a controlled temperature difference on the sample set by the controller, the furnace temperature is gradually ramped up or down to change the environment. When passing known phase transition zones, a slower heating rate was employed. TCs are connected to an Agilent 34970 data acquisition unit for simultaneous temperature and EMF measurement as shown in Fig. 1.

To apply the computation of Eq. 1 and quantify the measurement error, a piece of Chromel wire was first measured in the furnace. This Chromel wire, taken from a TC element, is not representative to the fuel pellet but has well-defined thermopower value for comparison. In this first measurement, the temperature difference between the two ends was not controlled, thus it varied from $5{ }^{\circ} \mathrm{C}$ to $30^{\circ} \mathrm{C}$ and changed sign. This represents extreme conditions that the later on measurements were not reached. $\mathrm{N}$ type TCs, in conjunction with the Chrome wire were used as reference material for the EMF measurement. The TC elements are all purchased from Omega. 
Three types of rod samples, having similar dimension to the fuel pellets, were measured for EMF. The first specimen was a $99.99 \%$ pure iron-nickel alloy that has a low transition temperature. Having the same dimensions, the second and third specimens were, respectively: $99.95 \%$ pure iron and a piece of commercial ANSI 1018 carbon steel. The high purity metal and alloys were purchased from ESPI Metals. The carbon steel was purchased from McMaster-Carr.

\section{Results and discussion}

Figure 3a presents the measured thermopower variation of Chromel with respect to change of temperature. For comparison, the values from literature are superimposed on the same figure. It indicates that the measured values closely follow the literature ones even with relatively large and variable $\Delta T$ from both the positive and negative legs of the $\mathrm{N}$ type TC (NP or NN) measurements. The two large spikes in measured thermopower near 200 ${ }^{\circ} \mathrm{C}$ are caused by the change of sign of temperature difference, i.e. the hot and cold ends of the specimen were reversed. With a temperature difference approaching zero, Eq. 1 is affected by the measurement uncertainty of the system. This figure shows the intrinsic merit of thermopower measurement: a function of material only.

Figure $3 \mathrm{~b}$ presents the deviation between the measured and reference values. The deviation is primarily less than $3 \%$. The deviation may be caused by uncertainty in the thermocouple which is $2.2{ }^{\circ} \mathrm{C}$ or $0.75 \%$ of reading, whichever is larger, or by the quasi-static measurement process because a true equilibrium may not be established. One observation that may be made about the deviation is that in the cooling process it is always slightly higher than in the heating process. This may be caused by thermocouple de-calibration which is the result of contamination causing thermal EMF drift in the thermocouples when exposed to high temperatures for long time. Another factor contributing to the deviation is the uncertainty and resolution of the data acquisition unit.

Using a controller to maintain a temperature difference of $\sim 5^{\circ} \mathrm{C}$ across the specimen, the temperature dependent EMF of iron-15 wt $\%$ nickel alloy was measured. Figure 4 presents the measured absolute thermopower of the alloy using both $\mathrm{N}$ and $\mathrm{S}$ type TCs. Some differences were shown from the two measurements at temperatures below $300{ }^{\circ} \mathrm{C}$ during the heating process. During the cooling process, the $\mathrm{N}$ type TC detached from the sample near $430^{\circ} \mathrm{C}$. The result from the $\mathrm{S}$ type TC (both negative, $\mathrm{N}$ and positive, $\mathrm{P}$ elements) was still able to reveal the sample's structure variation.

In the heating process, the Seebeck coefficient varied slowly with temperature change initially. However, the slope changed drastically close to $\sim 306-317^{\circ} \mathrm{C}$ for both TCs. This abrupt change of slope indicates that the alloy structure changed. A review of the Fe-Ni phase diagram [22] indicates that a eutectoid transformation occurs at 347 ${ }^{\circ} \mathrm{C}$. For $15 \mathrm{wt} \% \mathrm{Fe}-\mathrm{Ni}$ alloy, the combination of $\alpha \mathrm{Fe}, \mathrm{Ni}+\mathrm{FeNi}_{3}$ transforms to $\alpha \mathrm{Fe}, \mathrm{Ni}+\gamma \mathrm{Fe}$, Ni through a hypoeutectoid transformation. The difference of measured temperature from the literature one is due to the quasi-static measurement but a long holding time is necessary for the low temperature phase transition determination. Although slightly different from the equilibrium eutectoid temperature, this measurement technique demonstrates its capability of grasping material structure change even at such low temperatures.

Above the transition temperature, the measured Seebeck coefficients from both TCs increased with temperature at a similar rate until reaching another transition temperature. The inset in Figure 4 presents a close-up of the values from 590-740 ${ }^{\circ} \mathrm{C}$ where one can see that the measured transition temperature from the $\mathrm{N}$ type TC is approximately $609^{\circ} \mathrm{C}$ during the heating process. However, this temperature was measured to be around $630^{\circ} \mathrm{C}$ in the cooling stage. From the S type TC, the measured values for both heating and cooling were approximately 630 ${ }^{\circ} \mathrm{C}$. In the equilibrium phase diagram [22], the $\alpha \mathrm{Fe}, \mathrm{Ni}$ of this alloy converts completely to $\gamma \mathrm{Fe}, \mathrm{Ni}$ above $658^{\circ} \mathrm{C}$. Tanji [10] gives a measured solvus temperature as $635^{\circ} \mathrm{C}$. A comparison of the measured values with those from literature reveals good agreement. Another significant observation from these results is the measured values from $\mathrm{N}$ type TC varied largely above $600{ }^{\circ} \mathrm{C}$ while results from S type TC were quite consistent. Similar to Fig. 3, this may be due to de-calibration of the thermocouples; although the maximum recommended temperature for these diameter thermocouples is $870{ }^{\circ} \mathrm{C}$.

During the cooling process, the measured thermopower deviates from that of the heating process significantly. Thus the material structure of the alloy rod is not only influenced by its temperature but also by its heating history, the thermal lag of the instrument, and possibly differing kinetic rates for the forward and reverse reactions. In the $\gamma \mathrm{Fe}, \mathrm{Ni}$ temperature region, the holding time for the specimen temperature may not be long enough to transform its structure completely. For example, at a given temperature during the cooling process, the percentage of mixed phases may not be the same as that in the heating process. A much lower eutectoid temperature of approximately $200^{\circ} \mathrm{C}$ is observed in the cooling process and may be the temperature for completed transformation. 
At the conclusion of the run, the structure of the specimen changed back to its original state as reflected by the measured thermopower, which agreed with the initial measurement. Since the transition temperature is pretty low, and the diffusion at such low temperatures takes a very long time, there is certain error associated with the measurement. If the temperature changing rate is much slower, the phase change process will be more likely to complete, resulting in a more accurate measurement of phase transition temperature. Because the purpose was to show the ability of the system to determine phase change temperature, the experiment was not extended to a much longer time.

With the successful evaluation of material structure change for $\mathrm{Fe}-15 \mathrm{wt} \% \mathrm{Ni}$ at relatively low temperatures $\left(<800{ }^{\circ} \mathrm{C}\right)$, an effort was made to extend above $1000{ }^{\circ} \mathrm{C}$ using a pure iron sample. To improve stability, larger diameter TCs were employed. Figure 5 presents the measured thermopower of pure iron with respect to temperature. Positive thermopower was found at room temperature. The thermopower decreases to a negative value with an increase of temperature. However, above $500{ }^{\circ} \mathrm{C}$, the thermopower gradually increases until reaching the phase transition temperature. Around $913{ }^{\circ} \mathrm{C}$, the thermopower has an abrupt drop in the heating process from a positive value to negative. During the cooling process, the thermopower has a sharp rise around $910{ }^{\circ} \mathrm{C}$ and returns to a positive value. Above $950{ }^{\circ} \mathrm{C}$, the controller temporarily lost proper functionality causing a biased signal.

Because thermopower is an indication of material structure, it is apparent that a phase change occurs around $910-9133^{\circ} \mathrm{C}$. A comparison with the reference value of $912^{\circ} \mathrm{C}[22,23]$ at which the body-centered cubic (BCC) structure changes to face-centered cubic (FCC) or vice versa ( $\alpha \leftrightarrow \gamma$ transformation), shows that the experimental measurement was able to locate the transition temperature with good accuracy. During the heating process, slight overheating is needed for $\alpha$ phase to transform to $\gamma$ phase. During the cooling process, undercooling is required for FCC to return to BCC structure. Due to a complete phase transformation, the thermopower curve has a distinct discontinuity at the transformation temperature. This trend is different from that in Figure 4 where a change of slope occurs around the transition temperature due to a partial transformation. From this measurement, one can see that when the temperature change rate is very small and the transition temperature is relatively high, the quasi-static measurement can obtain a result very close to the equilibrium phase diagram.

In addition, at approximately $781^{\circ} \mathrm{C}$, the slopes of the measured thermopower change suddenly. This point is very close to the Curie point $\left(\mathrm{T}_{\mathrm{c}}\right)$ of $770{ }^{\circ} \mathrm{C}$ on the equilibrium phase diagram $[22,23]$ where a magnetic transformation occurs. The deviation between the measured and equilibrium values may be caused by a relatively fast heating/cooling rate in this region since the slower rate was only imposed on the phase transition temperature range. For the rest of the curve (room temperature to $600^{\circ} \mathrm{C}$ ), the thermopower in the cooling process is not identical to the one in the heating process probably because of thermal lag or some difference in material structure between the processes.

The third sample used for material structure evaluation was made of ANSI 1018 carbon steel. Even though it contains a small percent of manganese, phosphorus and sulfur, its structure can still be determined by the Fe-C phase diagram (according to the Fe-Mn phase diagram [24], the structure is $\alpha$-Fe in this weight percentage range). Figure 6 presents the measured thermopower for 1018 carbon steel.

Similar to that of pure iron, the thermopower evolves from a positive value at room temperature to a negative value. However, just as the trend turns positive, it hits the eutectoid transformation temperature at $725^{\circ} \mathrm{C}$. Since part of the ferrite and all the cementite transform to austenite, the slope of thermopower changes abruptly (see the inset). Above $849^{\circ} \mathrm{C}$, the remaining ferrite totally transforms to austenite causing another sudden slope change. In the cooling process, the $\delta \rightarrow \alpha$ transformation temperature does not differ significantly from the temperature in the heating process. However, noticeable supercooling is needed for the eutectoid transformation during the cooling process. Thus, around $672{ }^{\circ} \mathrm{C}$, the remaining austenite starts to transform back to pearlite. As indicated from the equilibrium metastable iron-cementite phase diagram [23], an eutectoid reaction occurs at $727^{\circ} \mathrm{C}$ where the hypoeutectoid pearlite transforms to a mixture of ferrite and austenite. For a carbon percentage of $0.15-0.2 \mathrm{wt} \%$ (ANSI 1018), the ferrite+austenite mixture entirely transforms to austenite at a temperature between $836-853^{\circ} \mathrm{C}$. Comparing the measured transition temperature values to the phase diagram, they are very close to those documented in literature.

\section{Conclusion}

An experimental system, capable of thermal conductivity and electromotive force measurements is described in this paper. EMF uncertainty evaluation was performed using Chromel from which the measured deviation is 
within 3-5\%. Calibration measurements on phase change materials were performed successfully. An iron-nickel alloy shows abrupt changes in measured EMF at certain temperatures corresponding to the phase transformation temperatures. The phase transition temperatures of a $99.95 \%$ pure iron sample were measured to be $910{ }^{\circ} \mathrm{C}$ and 913 ${ }^{\circ} \mathrm{C}$ in cooling and heating processes which tightly bounds the equilibrium temperature found in literature of $912{ }^{\circ} \mathrm{C}$. The Curie temperature was observed as well, but due to a relatively large temperature change rate, its value deviates slightly from the reported value. The measured phase transition temperatures for a multiple component alloy, ANSI 1018 carbon steel, closely follows the equilibrium metastable iron-cementite phase diagram. With the successful determination of material structure transitions for unitary, binary and multiple component alloys, this measurement system will be used to determine unknown material phase transformation temperatures.

\section{Acknowledgement}

The work is supported by U.S. Department of Energy, Office of Nuclear Energy, under DOE Idaho Operations Office, contract DE-AC07-05ID14517. Work performed by Colby Jensen is supported under a Department of Energy Nuclear Energy University Programs Graduate Fellowship.

\section{References}

[1] P.J. Spencer, A Brief History of CALPHAD, CALPHAD, 32 (2008) 1-8

[2] T. Ozawa, Thermal Analysis-Review and Prospect Thermochim. Acta, 355 (2000) 35-42

[3] W.H. Han, et al., Measurements of Vapor Pressure of Y and Activity of Y in Y-O Alloy by Multi-Knudsen Cell Mass Spectrometry, J. Alloy Compd., 481 (2009) 241-245

[4] E.G. Osadchii and D.A. Chareev, Thermodynamic Studies of Pyrrhotite-Pyrite Equilibria Ag-Fe-S System by Solid-State Galvanic Cell Technique at 518-723 K and Total Pressure of 1 atm, Geochim. Cosmochim. Ac., 70 (2006) 5617-5633

[5] T. Nakama, et al., Electrical Resistivity and Thermopower of ErCo3 under Hydrostatic Pressure, J. Phys.Conf. Ser., 150 (2009) 042136

[6] T.G. Ramesh and V. Shubha, Thermopower and Resistivity in the Study of Continuous Phase Transitions, in: Proceedings of the National Conference on High Pressure Science and Technology, Bangalore, India, 1994, pp. 6574.

[7] L.J. Li, et al., Superconductivity Induced by Ni Doping in BaFe2As2 Single Crystals, New J. Phys., 11 (2009) 025008(025001-025008)

[8] S.V. Ovsyannikov and V.V. Shchennikov, Phase Transitions Investigation in ZnTe by Thermoelectric Power Measurements at High Pressure Solid State Commun., 132 (2004) 333-336

[9] T. Tani and S. Tanaka, Thermoelectric Power Observation of Nearly-Commensurate Charge-Density Wave Phase in 1T-TaS2, J. Phys. Soc. Jpn., 53 (1984) 1790-1796

[10] Y. Tanji, et al., Anomalous Concentration Dependence of Thermoelectric Power of Fe-Ni (fcc) Alloys at High Temperatures, J. Phys. Soc. Jpn., 45 (1978) 1244-1248

[11] H. Wilhelm and D. Jaccard, Probing the Phase Diagram of Ce Ru2 Ge2 by Thermopower at High Pressure, Phys. Rev. B, 69 (2004) 214408 (214401-214406)

[12] C. Xing, et al., An Optimal Guarding Scheme for Thermal Conductivity Measurement Using a Guarded CutBar Technique, Part 1 Experimental Study, Appl. Therm. Eng., 62 (2014) 850-857

[13] C. Xing, et al., An Optimal Guarding Scheme for Thermal Conductivity Measurement Using a Guarded CutBar Technique, Part 2 Guarding Mechanism, Appl. Therm. Eng., 59 (2013) 504-514

[14] C. Folsom, et al., Experimental and Numerical Investigation on the Effective Thermal Conductivity of

Tristructural-Isotropic (TRISO) Fuel Compacts, J. Nucl. Mater., 458 (2015) 198-205

[15] C. Jensen, et al., Design and Validation of a High-Temperature Comparative Thermal-Conductivity Measurement System, Int. J. Thermophys., 33 (2012) 311-329

[16] R.D. Barnard, Thermoelectricity in Metals and Alloys, Taylor \& Francis Group, New York, 1972.

[17] ASTM, Standard Specification and Temperature-Electromotive Force (EMF) Tables for Standardized Thermocouples, 2003, E 230-03

[18] N. Causack and P. Kendall, The Absolute Scale of Thermoelectric Power at High Temperature, Proc. Phys. Soc., 72 (1958) 898-901

[19] R.B. Roberts, et al., Absolute Scale of Thermoelectricity III, Philos. Mag. B, 52 (1985) 1147-1163 
[20] K.P. Padmasree and D.K. Kanchan, Thermoelectric Power and Electrochemical Studies on CdI2-Ag2OV2O5-B2O3 System, J. Solid State Electrochem., 12 (2008) 1561-1565

[21] T. Bak, et al., Thermoelectric Power of Mixed Electronic-Ionic Conductors I. Basic Equations, Ionics, 10 (2004) 159-165

[22] L.J. Swartzendruber, et al., The Fe-Ni (Iron-Nickel) System, J. Phase Equilib., 12 (1991) 288-312

[23] H. Okamoto, The C-Fe (Carbon-Iron) System, J. Phase Equilib., 13 (1992) 543-565

[24] H. Okamoto, Desk Handbook: Phase Diagrams for Binary Alloys, ASM International, Materials park, OH, 2000. 


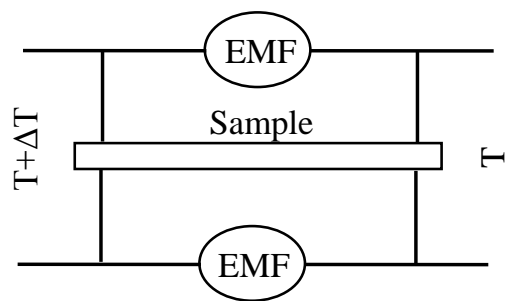

Figure 1 Schematic illustration for thermopower measurement

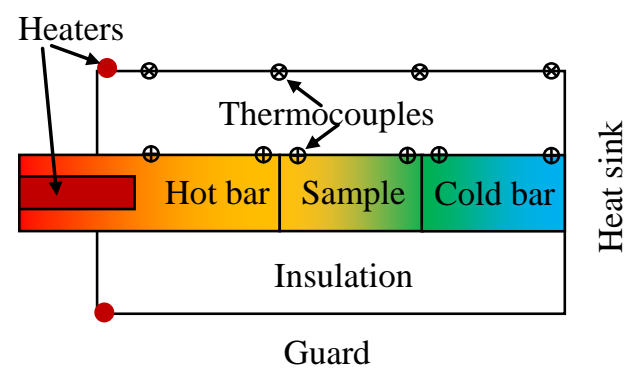

Fig. 2 Illustration of measurement system for thermal conductivity and thermopower
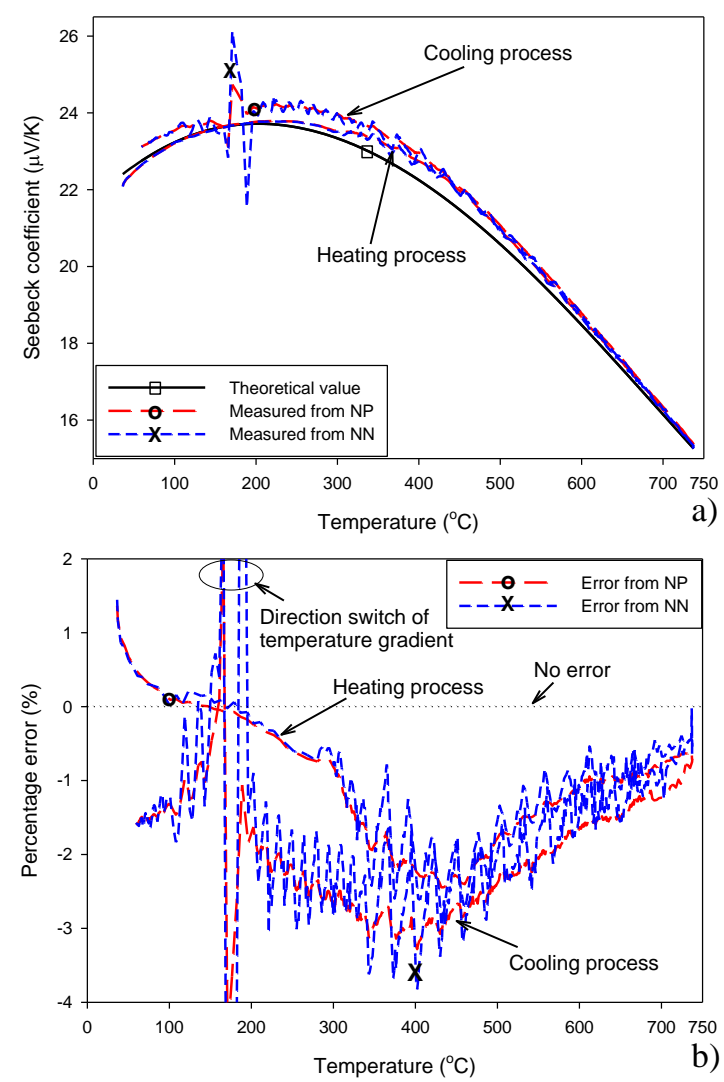

Fig. 3 Measured and literature Seebeck coefficients and the deviation of measured values from those in literature for Chromel a) Seebeck coefficient b) deviation 


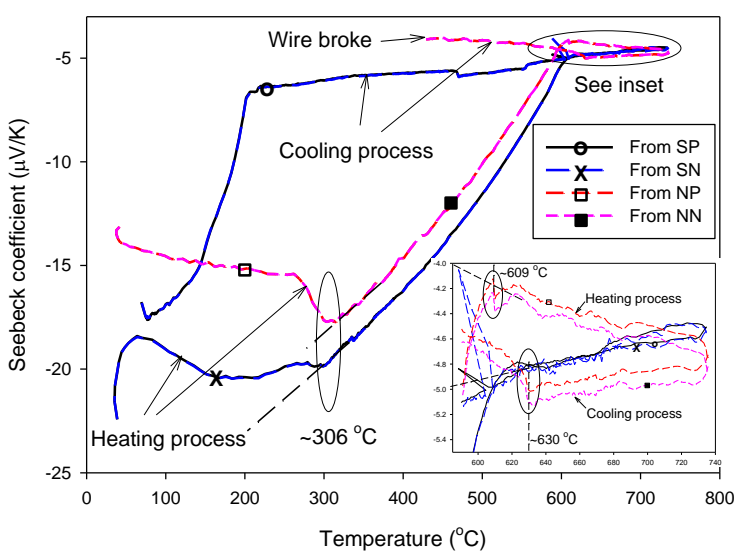

Fig. 4 Measured absolute thermopower of Fe- 15 wt $\%$ $\mathrm{Ni}$ alloy with respect to temperature. The inset presents a close-up of the values from $590-740{ }^{\circ} \mathrm{C}$

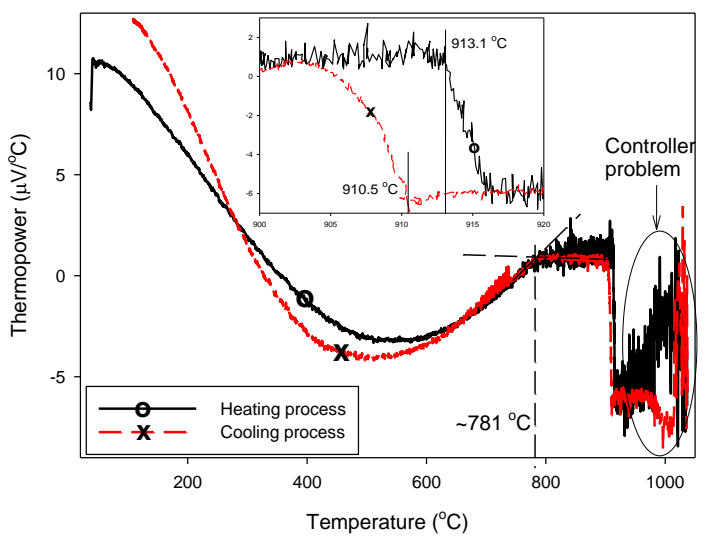

Fig. 5 Measured absolute thermopower of 99.95\% pure iron with respect to temperature

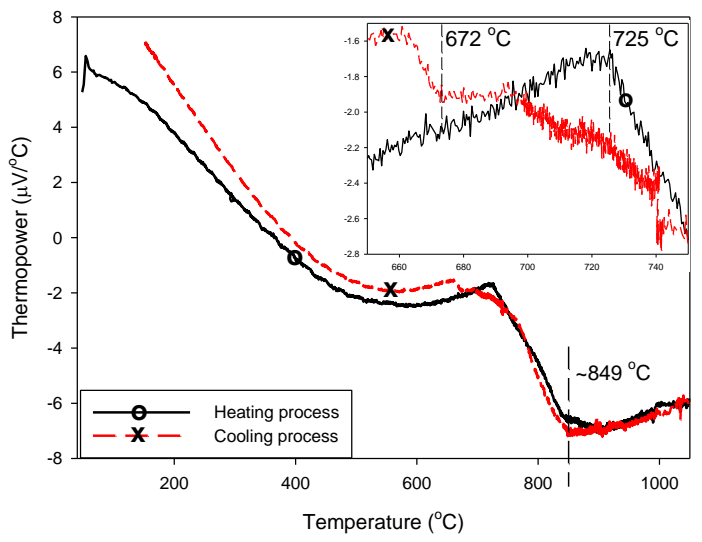

Fig. 6 Measured absolute thermopower of ANSI 1018 carbon steel with respect to temperature 